\title{
NOISES CONTROL AND ANALYSIS IN MEASURING DOMAIN SIZE
}

\author{
Z.-Y. LEE, P. ZHU, W.J. XIA, Y.S. HU, S.B. CHEN and X.S. MIAO \\ Dept. of Solid State Electronics, Huazhong Univ. of Sci. \& Tech., Wuhan, Hubei 430074, China
}

\begin{abstract}
For measuring a recorded domain size, the influence of noises may come from the fluctuation of light source and the CCD detector. By using a method of multiple accumulated domain image, these noises can be affectively controlled as the number of accumulated domain image approaching to a certain value. It has been found that the domain size is influenced by the illuminated light intensity, a description of a correctly choosing of the light intensity is stated. In order to reduce the fringing effect caused by the optical lenses, a method of one-third threshold greyscale is introduced. For calibrating the resolution of pixel, the calibrator is modified by electron microscopy.
\end{abstract}

\section{Introduction}

Amorphous rare earth-transition metal (RE-TM) thin films and newly developing multilayers $\mathrm{Pt} / \mathrm{Co}$ and $\mathrm{Pd} / \mathrm{Co}$ are proved to have high recording density. The recording density is directly related to domain size. [1,2]. So it is important to measure the domain size. A recorded domain is achieved and processed by our MO recording domain image processing and analysis system [3]. In this paper, we investigated the effects of noises caused by the fluctuation of light source, a CCD detector. These noises could be reduced by using a method of multiple accumulated domain image. The varying of light intensity and the fringing effect of objective lens, which have a effect on the measured results of domain size, are discussed.

\section{The Effect of Light Fluctuation and CCD Detector on Measurement}

Since the voltage for illuminated light is not stable, the intensity of light source would have a fluctuation, which is regarded as a kind of noise. Another is the noise produced by the CCD detector. They would vary the grey value of a domain image. Here we can use a method of multiple accumulated domain images to reduce them.

Table 1 the Measured domain diameters and its mean variance

\begin{tabular}{|c|c|c|c|c|c|c|c|c|c|c|}
\hline & \multicolumn{8}{|c|}{ doma in diameter $(\mu \mathrm{m})$} \\
\cline { 2 - 11 } & 1 & 2 & 3 & 4 & 5 & 6 & 7 & 8 & 9 & 10 \\
\hline 1 & 1.162 & 1.185 & 1.153 & 1.160 & 1.190 & 1.150 & 1.158 & 1.160 & 1.171 & 1.165 \\
\hline 10 & 1.163 & 1.170 & 1.165 & 1.154 & 1.158 & 1.171 & 1.160 & 1.176 & 1.159 & 1.166 \\
\hline mean variance & \multicolumn{7}{|c|}{$\sigma_{1}=0.013$} & \multicolumn{6}{c|}{$\sigma_{10}=0.005$} \\
\hline
\end{tabular}

where laser power, duration time and external field are $8 \mathrm{~mW}, 0.5 \mu \mathrm{s}$, and 2000 . 
Table 1 gives the results of domain diameter measured with two methods, where one is measured directly from the original domain, and the other is measured by accumulated ten original ones.

Fig.1 is a domain image accumulated with ten pictures of the original domain. The image seems to be sharp and clear.

Fig. 2 is the dependence of domain diameters on the number $N$ of accumulated domain image. The value of mean variance $\sigma$ is reduced and tends to a constant $\sigma_{c}$ with the number $N$ being increased. It shows that the noises caused by the fluctuation of light and the CCD detector are controlled.

on the other hand, that the light intensity influence on domain measurement is determined by no change of the boundary threshold greyscale, which is defined by the difference of maximum greyscale in center of a domain and the greyscale out of the domain.

\section{The Influence of Numerical Aperture of Objective Lens on Measurement}

It has been found that there is a distribution of greyscale in and out of a domain image, which is caused by the fringing effect of objective lens. In order to reduce this effect. one method is to increase the numerical aperture of objective lens, the other is adopted by one-third boundary threshold gryescale of the recorded domain image. The numerical aperture of objective lens in selected to be 0.65 . Thus, the error for measuring domain size is under 1\%. In our experiment a group of parallel lines is used as a calibrator for measuring domain size, because of convolution effect of objective lens for a small bit size, the final results is modified by the electron microscopy. The detail results will be shown in manuscript paper.

\section{Conclusion}

For measurement of domain size, the effects of several factors are investigated. The noises caused by the fluctuation of light source and the CCD detector could be reduced with a method of multiple accumulated original domain image. The light intensity is determined by no change of the reference boundary greyscale of a domain. The fringing effect caused by objective lens is decreased when its numerical aperture is selected to be large (Here, N.A. is 6.5). The error for measuring domain size is controlled under $1 \%$ by using one-third boundary threshold greyscale method. The convolution of optical lens for small bit size is discussed.

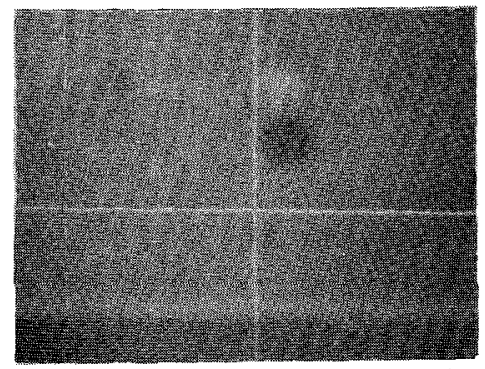

Fig.1 The photography of domain image accumulated with ten original domain

References:

[1] Han-Ping D. Shieh and Mark H. Kryder, J. Appl. Phys. 61(3), 1108(1987).

[2] M. Takahashi, T. Niihara, and N. Ohta, J. Appl. Phys. 64(1), 262(1988).

[3] Z. Y. Lee, et al, Jap. J. Appl. Phys. 28, Suppl. 28-3, 47(1989). 